Nevşehir Bilim ve Teknoloji Dergisi Cilt 6(ICAFOF 2017 Özel Sayı) 254-260 2017

DOI: 10.17100/nevbiltek.334595

URL: http://dx.doi.org/10.17100/nevbiltek.334595

\title{
Bazı Yabani Mantarların Antioksidan Özellikleri
}

\author{
S. Seçil ERDOĞAN ${ }^{1, *}$, Mustafa Kemal SOYLU ${ }^{2}$, Kemal Hüsnü Can BAŞER ${ }^{3}$ \\ ${ }^{1}$ Atatürk Bahçe Kültürleri Merkez Araştırma Enstitüsü, Gıda Teknolojileri Bölümü, Yalova \\ ${ }^{2}$ Atatürk Bahçe Kültürleri Merkez Araştırma Enstitüsü, Sebzecilik Bölümü, Yalova \\ ${ }^{3}$ Near East University, Faculty of Pharmacy, Department of Pharmacognosy, Lefkosa (Nicosia), N.
}

Cyprus, Mersin 10

Öz

Çalışmada Türkiye'de farklı bölgelerden toplanan 12 yabani mantarın (Lactarius piperatus, Tricholoma anatolicum, Amanita caesarea, Lactarius delicious, Lactarius sanguifluus, Cantharellus cibarius, Hydnum repandum, Picoa lefebvrei, Ramaria aurea, Lactarius semisanguifluuss, Craterellus cornucopioides, Laccaria laccata) antioksidan aktiviteleri ve özellikleri incelenmiștir. Toplanan mantarların methanolde elde edilen ekstrelerinde toplam fenolik madde miktarı Folin-Ciocalteu yöntemi, flavonoid miktarı kolorimetrik olarak, antioksidan yöntemi 2,2-Diphenyl-1-picrylhydrazyl (DPPH) yöntemi ile trolox eşdeğeri olarak belirlenmiştir. Mantarların toplam fenolik madde içeriği 575.10-2156.40 mg GAE/100g DW, flavonoit miktarı 103.01-346.53 mg CE/100g DW ve antioksidan aktivitesi 525.32 to $1693.85 \mu \mathrm{mol}$ (TE)/100 g DW arasında değişmiştir.

Anahtar Kelimeler: Yabani mantarlar, antioksidan aktivite, toplam fenolik, flavonoit

\section{Antioxidant Properties of Some Wild Mushrooms}

\section{Abstract}

The antioxidant activity and properties of 12 wild mushrooms (Lactarius piperatus, Tricholoma anatolicum, Amanita caesarea, Lactarius delicious, Lactarius sanguifluus, Cantharellus cibarius, Hydnum repandum, Picoa lefebvrei Ramaria aurea, Lactarius semisanguifluuss, Craterellus cornucopioides, Laccaria laccata) collected from Turkey were evaluated. Their methanolic extracts were used to determine antioxidant capacity (TAC), total phenolics and flavonoids. 2,2-Diphenyl-1-picrylhydrazyl (DPPH) radical scavenging activities were measured to evaluate antioxidant capacity of the extracts and expressed as trolox equivalents (TE). The amount of total phenolics was determined by using Folin-Ciocalteu method and Flavonoid contents in the extracts were determined by a colorimetric method. Wild mushrooms were found to be high in antioxidant phytochemicals, such as phenolics (575.10$2156.40 \mathrm{mg} \mathrm{GAE} / 100 \mathrm{~g}$ DW), flavonoids (103.01-310.89 mg CE/100g DW). The TAC values of the spices ranged from 525.32 to $1693.85 \mu \mathrm{mol}(\mathrm{TE}) / 100 \mathrm{~g}$ DW.

Keywords: Wild mushroom, methanolic extract, antioxidant activity, phenolic, flavonoid

*e-mail: seciley@hotmail.com 


\section{Nevşehir Bilim ve Teknoloji Dergisi Cilt 6(ICAFOF 2017 Özel Sayı) 254-260 2017}

1. Giriş

Yabani ve kültür mantarları farklı ve hoş lezzetleri ile geçmişten günümüze kadar insan beslenmesinin bir parçası olmuştur. Mantarlar protein, lif, vitamin ve mineral zenginliğine ek olarak birçok ülkede geleneksel tıpta kullanılmıştır [1]. Ülkemiz mantar türleri bakımından oldukça zengindir. Birçok mantar türü ülkemizden taze, soğutulmuş, dondurulmuş, salamura ve kurutulmuş olarak ihraç edilmektedir. Yılda 1.813 ton doğal ve kültür mantarı ihraç edilmektedir ve bunların \%99,5'i ektomikorizal mantarlardır. Bu ihracatın toplam ekonomik değeri ise yaklaşık 13 milyon Amerikan dolarıdır. Lactarius deliciosus (L.) Gray, Craterellus cornucopioides (L.) Pers, Amanita caesarea (Scop.) Pers, Hydnum repandum L., Cantharellus cibarius Fr. ihraç edilen türlerden bazılardır [2].

Yaşanılan çevrede yüksek miktarlarda oksidatif etkenler vardır ve metabolizma dahil birçok süreç bu ortamdaki oksidanların artmasına neden olur. İnsanların ve hayvanların karmaşık bir antioksidan savunma sistemleri vardır ancak yine de bu sistem mükemmel değildir ve oksidatif zarar meydana gelir. Özellikle kardiyovasküler hastalıkların ve kanserin oksidatif stresten meydana geldiği düşünülmektedir [3]. Antioksidanların oksidatif stres sonucu oluşan dejeneratif ve yaşla ilgili çeşitli hastalıkları önlemedeki rolü deneysel, klinik ve epidemiyolojik çalışmalar ile ortaya konmaya başlandıkça antioksidanlar gittikçe daha çok önem kazanmaya başlamıştır [4]. Mantarlar içerdiği polifenolik bileşikler sayesinde, tek elektron transferi ile serbest radikalleri temizleme kabiliyetlerinden ötürü mükemmel bir antioksidandır [5]. Farklı mantar türleri arasında araştırmacılar tarafından antioksidan aktivite ve fenolik bileşenlerine ait yapılmış pek çok çalışma bulunmaktadır [5-10]. Çoğu çalışmada yenen mantarların güçlü antioksidan aktiviteye sahip olduğu sonucuna varılmıştır. Mantarlarda bulunan antioksidanlar özellikle fenolik asitler ve flavonoitlerdir [11].

Yapılan bu çalışmada hepsi yenebilen Lactarius sanguifluus (Mor kanlıca) Yalova fistık çamı ormanından, Cantharellus cibarius (Sarı kız, kaz ayağı) Bolu kayın ormanından, Picoa lefebvrei (Kara keme) Şanlıurfa-Tülmen mera alanından, Craterellus cornucopioides (Borozan mantarı) Yalova Güneyköy kayın ormanından, Amanita caesarea (İmparator mantarı) Tekirdağ-Saray kayın ormanından, Lactarius piperatus (Acı mantar) Yalova-Gacık Köyü kayın ormanından, Tricholoma anatolicum (Sedir, katran mantarı) Adana-Feke sedir ormanından, Hydnum repandum (Sığır dili) Yalova-Kurtköy kayın ormanından, Lactarius deliciosus (Kanlıca, melki, çıntar) Yalova-Armutlu-Selimiye köyü çam ormanından, Lactarius semisanguifluus (Mor kanlıca) Yalova-Kabaklı köyü çam ormanından, Laccaria laccata Yalova-Merkez fistık çamı ormanından, Ramaria aurea (Karnabahar, yüzük mantarı) YalovaSoğucak kayın ormanından toplanarak toplam fenolik, flavonoit miktarları ve antioksidan aktiviteleri belirlenmiştir.

\section{Materyal ve Metot}

$\mathrm{Bu}$ çalışmada kullanılan mantarlar; Lactarius piperatus, Tricholoma anatolicum, Amanita caesarea, Lactarius delicious, Lactarius sanguifluus, Cantharellus cibarius, Hydnum repandum, Picoa lefebvrei, Ramaria aurea, Lactarius semisanguifluuss, Craterellus cornucopioides, Laccaria türleridir. Toplandıktan sonra laboratuvara getirilen mantarlar Heto CD 4 tipi liyofilizatörde dondurularak kurutulmuştur. 
Örneklerin ekstraksiyonu; dondurularak kurutulmuş örnekten alınan 1.5 g materyal $40 \mathrm{ml}$ metanolle $25^{\circ} \mathrm{C}^{\prime}$ de $150 \mathrm{rpm}$ 'de 12 saat çalkalanmış ve Whatman No. 4 süzgeç kağıdından süzülmüştür. Kalan kısım $20 \mathrm{ml}$ metanolle 4 saat tekrar çalkalanarak süzülen özütler toplanmıştır. Toplanan metanol özütünün $40^{\circ} \mathrm{C}$ 'de rotary evaparatörde metanolü uçurulduktan sonar kalan kurumuş kalıntı metanolle 50 ml'ye tamamlanmış ve analiz anına kadar $4{ }^{\circ} \mathrm{C}$ 'de muhafaza edilmiştir [10].

Antioksidan aktivite 2,2-Diphenyl-1-picrylhydrazyl (DPPH) radikal süpürücü metodu kullanılarak belirlenmiştir [12, 13]. Özütten alınan $5 \mathrm{ml}$ örneğin üzerine \% 0.004'lük metanollü DPPH solüsyonu eklenmiş ve 30 dakika karanlık ve oda koşullarında bekledikten sonra $517 \mathrm{~nm}$ de spektrofotometrede de okunmuştur. Denemeler üç tekerrürlü olarak yapılmış ve 100 gram kuru ağırlık üzerinden $1 \mathrm{M}$ troloks eşdeğeri (TE) olarak ifade edilmiştir.

Toplam fenolik bileşikler Folin-Ciocalteu metodu ile tespit edilmiştir [9]. $1 \mathrm{ml}$ ekstre ile $1 \mathrm{ml}$ Folin-Ciocalteu ayıracı karıştırılmış 3 dakika sonra $1 \mathrm{ml}$ sodyum karbonat eklenerek distile su ile $10 \mathrm{ml}$ tamamlanmıştır. 90 dakika karanlıkta bekletildikten sonra $725 \mathrm{~nm}$ dalga boyunda okuma yapılmıştır. Sonuçlar gallik asit ile hazırlanan eğri ile mg gallik asit eşdeğeri (GAE) / 100 g KM olarak ifade edilmiştir.

Flavonoit miktarı kolorimetrik metot ile belirlenmiştir. $0.5 \mathrm{ml}$ ekstrenin üzerine $2 \mathrm{ml}$ distile su eklendikten sonra $0.15 \mathrm{ml} \%$ 15’lik $\mathrm{NaNO}_{2}, 6$ dakika sonra $0.15 \mathrm{ml} \% 10 \mathrm{AlCl}_{3}$ eklenmiş, 6 dakika sonra $2 \mathrm{ml} \% 4$ 'lük $\mathrm{NaOH}$ solüsyonu eklenerek örnek hacmi distile su ile 5 ml'ye tamamlanmıştır. 15 dakika sonra $510 \mathrm{~nm}$ dalga boyunda ölçüm yapılmıştır. (+)-kateşin kullanarak hazırlanan eğriye göre miktarlar hesaplanarak sonuçlar mg kateşin eşdeğeri (KE) / 100 g KM olarak verilmiştir [14]. Sonuçların değerlendirilmesinde varyans analizi uygulanmış, önemli bulunanlara asgari önemli fark testi uygulanarak \%5 düzeyinde gruplandırma yapılmıştır [15].

\section{Bulgular ve Tartışma}

Yapılan analizler sonucu elde edilen bulgular aşağıda verilmiştir. Buna göre antioksidan aktivite toplam fenol ve flavonoit miktarları Tablo 1'de verilmiştir.

İstatistik değerlendirme sonucunda, antioksidan aktivite en yüksek $H$. repandum mantar türünde belirlenirken 12 mantar türü antioksidan aktivite, toplam fenolik ve flavonoit bazında kendi aralarında dört gruba kategorize edilebilir; çok yüksek, yüksek, orta ve düşük olarak. Buna göre H.repandum, R. aurea, L. deliciosus çok yüksek, C.cornucopioides, L. sanguifluus, L. Piperatus, yüksek, P. lefebvrei, T. anatolicum, C. Cibarius, A.caesarea orta, L. laccata ve L. semisanguifluuss düşük olarak sınıflandırılmıştır. Toplam fenolik madde arasında yapılan sınıflandırmada; $R$. aurea çok yüksek, C. cornucopioides, L. semisanguifluuss, A.caesarea, L. delicious ve H. repandum yüksek, C. cibarius, L. piperatus, L. sanguifluus, Laccaria laccata orta, P. lefebvrei ve T. Anatolicum düşük olarak sınıflandırılmıştır. Flavonoid madde miktarında yapılan sınıflandırmada; L. semisanguifluuss çok yüksek, L.piperatus, P.lefebvrei ve C.cornucopioides yüksek, R.aurea ve L.s delicious orta, L. Laccata, H. repandum C. cibarius, A.caesarea ve T. anatolicum düşük olarak sınıflandırılmıştır. Antioksidan aktivite ile toplam fenol ve flavonoit miktarı arasında korelasyon belirlenememiştir. 
Nevşehir Bilim ve Teknoloji Dergisi Cilt 6(ICAFOF 2017 Özel Sayı) 254-260 2017

Tablo 1. Mantar türleri arasında antioksidan aktivite, toplam fenolik madde ve flavonoid miktarı

\begin{tabular}{|c|c|c|c|}
\hline \multirow{2}{*}{ Mantar türleri } & Antioksidan aktivite* & Toplam fenolik madde** & Flavonoit miktarı**** \\
\hline & ( $\mu \mathrm{mol}$ TE / 100 g KM) & (mg GAE / 100 g KM) & (mg KE / 100 g KM) \\
\hline Lactarius piperatus & $* * * * 1266,95 \pm 13,03 \mathrm{~d}$ & $1213,53 \pm 55,07 \mathrm{~d}$ & $285,93 \pm 51,15 \mathrm{ab}$ \\
\hline Tricholoma anatolicum & $936,00 \pm 41,89 \mathrm{f}$ & $575,10 \pm 29,49 \mathrm{~g}$ & $131,12 \pm 16,60 \mathrm{~d}$ \\
\hline Amanita caesarea & $858,85 \pm 11,36 \mathrm{~g}$ & $1453,95 \pm 45,19$ c & $103,01 \pm 10,24 \mathrm{~d}$ \\
\hline Lactarius sanguifluus & $1283,51 \pm 8,43 \mathrm{~cd}$ & $1133,12 \pm 29,90$ e & $253,98 \pm 7,10 \mathrm{~b}$ \\
\hline Lactarius deliciosus & $1610,18 \pm 1,47 \mathrm{~b}$ & $1451,51 \pm 23,34$ c & $173,36 \pm 19,44$ c \\
\hline Cantharellus cibarius & $848,85 \pm 49,44 \mathrm{~g}$ & $1277,77 \pm 23,51 \mathrm{~d}$ & $112,56 \pm 18,79 \mathrm{~d}$ \\
\hline Hydnum repandum & $1693,85 \pm 12,30 \mathrm{a}$ & $1409,39 \pm 86,70 \mathrm{c}$ & $107,87 \pm 12,97 \mathrm{~d}$ \\
\hline Picoa lefebvrei & $1030,66 \pm 43,48$ e & $708,56 \pm 38,71 \mathrm{f}$ & $272,20 \pm 29,01 \mathrm{ab}$ \\
\hline Ramaria aurea & $1612,99 \pm 2,89 \mathrm{~b}$ & $2156,40 \pm 66,23 \mathrm{a}$ & $180,30 \pm 26,63$ c \\
\hline Lactarius semisanguifluuss & $525,32 \pm 28,111$ & $1460,47 \pm 34,75$ bc & $310,89 \pm 37,00 \mathrm{a}$ \\
\hline Craterellus cornucopioides & $1319,11 \pm 22,28$ с & $1529,79 \pm 32,17 \mathrm{~b}$ & $256,56 \pm 9,98 \mathrm{~b}$ \\
\hline Laccaria laccata & $710,83 \pm 14,95 \mathrm{~h}$ & $1080,10 \pm 4,99 \mathrm{e}$ & $120,14 \pm 19,52 \mathrm{~d}$ \\
\hline
\end{tabular}

*Antioksidan aktivite değerlerinin sınıflandırılması; çok yüksek $>1500$, yüksek=1500-1200, orta=1200-700, düşük<700 $\mu$ mol TE / $100 \mathrm{~g} \mathrm{KM}$

**Toplam fenolik madde miktarının sınıflandırılması; çok yüksek >1500, yüksek=1500-1300, orta=1300-1000, düşük<1000 mg GAE / 100 g KM

***Flavonoit miktarının türler arasında sınıflandırılması; çok yüksek > 300, yüksek=300-250, orta=250-150, düşük<150 mg KE / 100 g KM

****Her değer üç tekrarlı tespitlerin ortalamasıdır. Farklılıkları önemli bulunanlar harflendirilmiştir $(\mathrm{p}<0.05)$.

Yapılan farklı çalışmalarda; Ramirez-Anguiano, Ribeiro, Barros ve arkadaşlarının atfen bildirdiğine göre en yaygın tüketilen $C$. cibarius türü $B$. edulis ve L. giganteus türleri en düşük antioksidan aktivite göstermiştir [16]. Yaptığımız çalışmada da C. cibarius düşük antioksidan aktivite gösteren türler arasında yer almıştır. Heleno ve ark.'nın yaptıkları çalışmada H. repandum, L.laccata, L. salmonicolor türlerinde antioksidan aktivite değerinde $H$. repandum en yüksek $\mathrm{EC}_{50}$ değerini verirken onu L. laccata ve L. salmonicolor türleri takip etmiştir [9]. Çalışmamızla aynı şekilde toplam fenolik madde ile antioksidan aktivite arasında korelasyon olmadığı gözlemlenmektedir. Palacious ve ark. farklı mantar türlerinin metanol ekstrelerini, antioksidan aktivite yönünden inceledikleri araştırmada C. cibarius (\%74) ve C. cornucopioides (\%70) en yüksek inhibasyonu gösterirken bu türleri L. deliciosus (\%50) takip etmiştir [17]. Kalogeropoulos ve ark. DPPH yöntemi ile antioksidan aktiviteyi L.dDeliciosus mantar

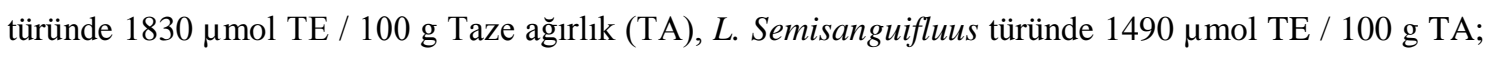
FRAP yönteminde L. deliciosus türünde $1320 \mu$ mol TE / 100 g TA, L. semisanguifluus'da $1700 \mu$ mol TE / 100 g TA olarak belirlemişlerdir [18]. Çetin yaptığı çalışmada C. cibarius mantar türünde \% DPPH· radikali giderme aktivitesinin L. volemus ve M. elata türlerine göre daha düşük olduğunu belirtmiştir [19]. Sarıkürkçü ve ark. A. caesarea türünün DPPH radikalini süpürücü yeteneğini (\%79.4) C. geotropa ve $L$. pudicus mantar türlerine göre daha yüksek olduğunu tespit etmiştir [20].

Ferreira ve ark. L. deliciosus mantar türünde toplam fenolik maddeyi 1066 mg / 100 g [8], Orhan ve Üstün L. deliciosus'da toplam fenolik maddeyi 5127 mg GAE / 100 g olarak, C. cibarius mantar türünde ise $3148 \mathrm{mg} \mathrm{GAE} \mathrm{/} 100 \mathrm{~g}$ tespit etmişlerdir. Aynı çalışmada antioksidan aktivite yönünden $L$. 
deliciosus, C. cibarius' dan ve diğer mantar türlerinden daha yüksek antioksidan aktivite göstermiştir [21]. Puttaraju ve ark. L. deliciosus'in su ekstresinde ve metanol ekstresinde sirasiyla toplam fenolik maddeyi 740 mg GAE / 100 g ve 480 mg GAE / 100 g, H. repandum'da 740 mg GAE / 100 g ve 260 mg GAE / 100 g, C. cibarius'da 200 mg GAE / 100 g ve 280 mg GAE / 100 g, L.sanguifluus 1780 mg GAE / 100 g ve 730 mg GAE / 100 g olarak belirlerken, antioksidan aktivite yönünden L. deliciosus’un diğer üç tipe göre daha yüksek antioksidan aktivite gösterdiğini belirtmişlerdir [22]. Heleno ve ark.'nın yaptıkları çalışmada taze ağırlık üzerinden toplam fenolik maddeyi L. salmonicolor türünde $414 \mathrm{mg}$ GAE / 100g, H. repandum'da $50 \mathrm{mg}$ GAE / 100g, L. laccata türünde ise $159 \mathrm{mg}$ GAE / 100g olarak tespit etmişlerdir [8]. Palacios ve ark.'nın yaptıkları çalışmada ise toplam fenolik madde miktarı büyükten küçüğe, $C$. cibarius, C. cornucopioides, L. deliciosus sıralamasında olmuştur [17]. Kalogeropoulos ve ark. yaptıkları çalışmada L. deliciosus mantar türünde toplam fenolik içeriğini, taze ağırlık esas alınarak, 12.4 mg GAE / 100g belirlerken, L. semisanguifluus türünde 10.412 .4 mg GAE / 100 g tespit etmişlerdir [18]. Çalışmada yapılan diğer çalışmaların ve bizim yaptığımız çalışmanın aksine toplam fenolik madde ile DPPH ve FRAP yöntemi ile antioksidan aktivite tayinleri arasında korelasyon olduğu belirtilirken aynı çalışmada metal şelatlama yönteminde ise korelasyon göstermediği belirtilmiştir.

Palacios ve ark.'nın yaptıkları çalışmada flavonoit madde miktarı en fazla L. deliciosus, mantar türünde belirlenirken bunu C. cornucopioides, C. cibarius takip etmiştir. Çalışmada flavonoit madde miktarı ve toplam fenolik madde arasında korelasyon olmadığı belirtilmiştir [7].

\section{Sonuç}

Bugüne kadar pek çok araştırıcı tarafından yapılan araştırmalarda mantarların antioksidan aktivitesinin yüksek olduğu belirtilmiştir. Mantar ekstrelerinin antioksidan aktivitesinin muhtemel mekanizması hidrojen bağlama kapasitesi ve peroksidaz radikallerinin oksidasyonu yoluyla meydana gelmektedir. Çalışmamızda da Türkiye'nin farklı bölgelerinden toplanan yabani mantarların antioksidan aktivitelerinin yüksek olduğunu tespit ettik. Yabani mantarların antioksidan özelliklerinin yüksek olması bu mantarlara olan ilgiyi günden güne artırmıştır. Bu nedenle hem iç tüketim hem de ihracatta her geçen yıl tüketim artmaktadır. Ancak bu konunun daha fazla çalışılması, mantar türlerine ait sağlık yönünden önemli unsurların ileride yapılan araştırmalarla incelenmesi önemlidir.

\section{Kaynaklar}

[1] Manzi P., Gambelli L., Marconi S., Vivanti V., Pizzoferrato L., "Nutrients in edible mushrooms: an interspecies comparative study.” Food Chemistry, 65, 477-482, 1999

[2 ] Soylu M.K., Öztürk M., Karik Ü., Boztok K., "Yenilebilir Doğal ve Kültür Mantarlarının Ülkemiz Dış Ticaretindeki Önemi” Türkiye VIII. Sebze Tarımı Sempozyumu, s.522-526, Van, 2010

[3] Ames B, Mark KS, Hagen TM., "Oxidants, Antioxidants, and The Degenerative Diseases of Aging” Proc. Natl. Acad. Sci. USA, Vol. 90, pp. 7915-7922, 1993

[4] Can A, Özçelik B, Güneş G., Meyve Sebzelerin Antioksidan Kapasiteleri. GAP IV. Tarim Kongresi, 1458-1461, Şanliurfa, 2005 
[5] Barros L., Ferreira I.C.F.R, Queiro’s B., Ferreira I., Paula Baptista "Total phenols, ascorbic acid, b-carotene and lycopene in Portuguese wild edible mushrooms and their antioxidant activities" Food Chemistry, 103, 413-419, 2007

[6] Leong L.P., Shui G., "An investigation of antioxidant capacity of fruits in Singapore markets" Food Chemistry, 76, 69-75, 2002

[7] Yanga J-H., Linb H-C., Maub J-L., “Antioxidant properties of several commercial mushrooms” Food Chemistry, 77, 229-235, 2002

[8] Ferreira I.C.F.R., Baptista P., Vilas-Boas M., Barros L., "Free-radical scavenging capacity and reducing power of wild edible mushrooms from northeast Portugal: Individual cap and stipe activity" Food Chemistry, 100, 1511-1516, 2007

[9] Heleno S. A., Barros L., Sousa M.J., Martins A., Ferreira I.C.F.R., "Tocopherols composition of Portuguese wild mushrooms with antioxidant capacity” Food Chemistry 119, 1443-1450, 2010

[10] Wootton-Beard P.C., Moran A., "Stability of the total antioxidant capacity and total polyphenol content of 23 commercially available vegetable juices before and after in vitro digestion measured by FRAP, DPPH, ABTS and Folin-Ciocalteu methods” Lisa Ryan Food Research International 44, 217-224, 2011

[11] Vaz J. A., Heleno S. A., Martins A., Almeida G.M., Vasconcelos M.H., Ferreira I.C.F.R., "Wild mushrooms Clitocybe alexandri and Lepista inversa: In vitro antioxidant activity and growth inhibition of human tumour cell lines” Food and Chemical Toxicology, 48 , 2881-2884, 2010

[12] Burits M. and Bucar F., “Antioxidant activity of Nigella sativa essential oil” Phytotheraphy Research, 14, 323-328, 2000

[13] Wojdyło A, Oszmian'ski J., Czemerys R., "Antioxidant activity and phenolic compounds in 32 selected herbs” Food Chemistry, 105, 940-949, 2007

[14] Asadi S., Ahmadiani A., Esmaeili M.A., Sonboli A., Ansari N., Khodagholi F., "In vitro antioxidant activities and an investigation of neuroprotection by six Salvia species from Iran: A comparative study” Food and Chemical Toxicology, 48, 341-1349, 2010

[15] Kalaycı M., "Örneklerde jump kullanımı ve tarımsal araştırma için varyans analiz modelleri” Anadolu tarımsal araştırma enstitüsü müdürlü̆g̈u yayınları, yayın no 21, Eskişehir, 296 syf., 2005

[16] Kalač P., "Chemical composition and nutritional value of European species of wild growing mushrooms: A review” Food Chemistry, 113, 9-16, 2009

[17] Palacios I., Lozano M., Moro C., D’Arrigo M., Rostagno M.A., Martínez J.A., García-Lafuente A.,Guillamón E., Villares A., "Antioxidant properties of phenolic compounds occurring in edible mushrooms” Food Chemistry, 128, 674-678, 2011

[18] Kalogeropoulos N., Yanni A.E., Koutrotsios G., Aloupi M., "Bioactive microconstituents and antioxidant properties of wild edible mushrooms from the island of Lesvos, Greece" Food and Chemical Toxicology, 55, 378-385, 2013

[19] Çetin F., "Tokat Bölgesinde Yetişen Bazı Yenebilen Yabani Mantar Türlerinde Yağ Asidi Kompozisyonları ve Antioksidan Kapasitelerinin Araştırılması” Gaziosmanpaşa Üniversitesi, Yüksek. Lisans Tezi, 61s, Tokat, 1992 
[20] Sarikurkcu C., Tepe B., Karslı Semiz D., Solak M.H., "Evaluation of metal concentration and antioxidant activity of three edible mushrooms from Mugla, Turkey" Food and Chemical Toxicology, 48, 1230-1233, 2010

[21] Orhan i., Üstün O., "Determination of total phenol content, antioxidant activity and acetylcholinesterase inhibition in selected mushrooms from Turkey" Journal of Food Composition and Analysis 24, 386-390, 2011

[22] Puttaraju, N. G., Venkateshaiah, S. U., Dharmesh, S. M., Urs, S. M. N., \& Somasundaram, R., “Antioxidant activity of indigenous edible mushrooms” Journal of Agricultural and Food Chemistry, 54, 9764-9772, 2006 